\title{
TRANSLATING GRAFCET SPECIFICATIONS INTO C/UNIX PROGRAM
}

\author{
Nacéra Benaouda and Abdelhafid Benaouda \\ SETIF-1 University, Algeria
}

\begin{abstract}
This paper presents a method of simulating the operation of the control part of an automated system, modeled by a Grafcet. Our approach is to translate the Grafcet into C language, in a Unix environment. The resulting program simulates the operation of the automation in question. The first step is to establish the Grafcet / C generation schemes. A Grafcet put in internal form will be translated in $\mathrm{C}$ by a generating program, on the basis of the schemes previously found. This will allow the validation of the functional specifications of a sequential automation as, for educational purposes, the learning of the Grafcet formalism. Once the program has been configured, tested and validated, the object code can be implemented on a microcontroller of the actual control system.
\end{abstract}

\section{KEYWORDS}

Grafcet, Process, C/Unix

\section{INTRODUCTION}

Equipment manufacturers and automation engineers responsible for automated industrial installations must be able to understand the programs that drive their systems, in order to be able to carry out their corrective, adaptive or evolutionary maintenance missions (Benaouda. N, 2017). They want to do it without having to (re) program, especially since these programs often under treated, could use a variety of languages and libraries, depending as much on their provider's past as on the problem to be solved. At the programming languages Babel Tower, automation experts oppose the desire for specification formalisms, allowing them to dominate the situation, while expressing for each functionality all that they consider necessary, and nothing more.

In this work, we focus on sequential automated systems, where the control part is modeled by the Grafcet tool (Blanchard..M, 1994), (Reeb.B, Chèze, 2011). In an automated system, the control part is nothing else than the image of the operative part which concretely represents the automated machine. Before implementing the automation on actual installations, the simulation and validation of its operation is necessary. Simulating the operation of Grafcet amounts to translating it into appropriate languages which generate programs with the same semantics. These programs have shown their usefulness at several levels, namely, the validation of the design before the implementation of the automation on actual installations, in industrial maintenance tasks, with an educational aim for learning the Grafcet formalism, and also, once the program is configured, tested and validated, the object code can be implemented on the microcontroller of the control system itself. In our work, we have chosen a translation of Grafcet in $\mathrm{C}$ language in a Unix environment. The $\mathrm{C}$ language extended by the Unix library has all tools allowing to translate Grafcet, moreover, the required configuration for the realization is very simple: a personal computer with a linux distribution. To illustrate our work we begin by presenting some related works, showing their advantages and disadvantages. We then present our target language, $\mathrm{C}$ under Unix, emphasizing the tools for creating processes, communication between processes and timings. The following section contains a description of our simulation system. We continue with section 5 in which we present our approach in detail, by proposing an original approach that allowed us to develop the translation schemes of Grafcet in $\mathrm{C}$. We end with a conclusion that encompasses the advantages and peculiarities of our work as well as a relevant perspective. 


\section{RELATED WORK}

A big number of research work have been achieved to formalise the Grafcet. This research date back further, like (Remaki. Z and al., 1994), or in recent years such as (Gonzalez. E and al., 2016), (Julius.R and al., 2017), (Julius. R and al., 2019) and (Miguel-Escrig.O et al, 2020).

(Remaki.Z and al., 1994) have designed the translation schemes of the grafcet in Occam2 language which is executable on transputers. The authors have exploited the possibilities of expressions of parralel tasks offered in Occam 2 to express the parallel tasks represented in the grafcet. The resulting program could be run on a parallel machine and simulated the actual operation of an automated system. With this work, we could from a grafcet in graphical or textual form obtain the equivalent Occam2 program that simulates the operation of the automation. By being a parallel language, Occam 2 possesses the different tools that can translate a grafcet; but the resulting program can only be exploited if a parallel machine existed. Since parallel machines are only available in certain research laboratories or in specific industrial settings, such a program may have limited use.

(Gonzalez.E and al., 2016) have defined a semi-coarse ontology that they wrote in the web language of ontology. To test it, they integrated it into an already existing educational tool to teach Grafcet intended for use in programmable logic controllers. This ontology is OWL (Web Ontology Language) DL() based, a specific decidable fragment of first-order logic which is applied to OWL markup language. The objective of this method is to give a complement to other propositions previously defined by other authors, and in the same time to promote this type of techniques in the formalisation of the grafcet. Advantages of ontologies are numerous, they make the sharing of knowledge and the collaboration easier, they guarantee greater reliability, more over they assure to handle automatically any input change without having to recompile the processing code. The validation of the approach defined in this work was realized according to two criteria, accuracy and completeness.

(Julius.R and al, 2017) have defined an approach that automatically generates PLC (Programmable Logic Controller) code corresponding to a GRAFCET specification, with ST (Structured Text) as the target command programming language. The initial hierarchical structure of Grafcet is preserved by the resulting control program, and this without requiring standardization, as in the case of previous approaches. To avoid ambiguity in the interpretation of Grafcet dependency elements, the notion of hierarchical elements was used to extend the interpretation algorithm for Grafcet on sequential systems. The validation of the approach was carried out by making a comparison of the structure, the content and the behavior of the code generated automatically according to the adopted approach, with the code which had been implemented by human programmers of automatons and which are based strictly on the Grafcet specification.

The work presented in (Julius.R and al, 2019) can be considered as an extension of that presented in (Julius.R et al. 2017). It is always the automated generation of PLC code, from a Grafcet specification, and always having ST as target language, while preserving the initial hierarchical structure of Grafcet. But this time around, the approach is based on a model. The system produced includes a Grafcet editor and a rule-based ST code generator. Compared to (Julius. R and al. 2017), (Julius.R and al, 2019) includes temporal dependencies, and has been implemented using a model-based approach in the Eclipse environment via EMF and Acceleo. The authors presented a sample application to demonstrate the usability and accuracy of their approach.

Within (Miguel-Escrig. O et al, 2020) a new vision was adopted. From a Grafcet, the authors proposed the systematic implementation of the control software in IEC 61499. This constitutes a key advantage over previous Grafcet implementations, because it allows engineers to implement models of Grafcet, which are distributed over several devices, and at the same it keeps the initial centralized design. IEC 61499 has all the translation tools for most of the Grafcet elements. This work makes it possible to systematically translate grafcet and introduces several translation models. The disadvantage in IEC 61499 lies in the fact that it is not possible to model the structuring mechanisms such as fences or forcing steps, moreover, the macros-steps that can be implemented are limited to simple sequences. The authors presented an example of application in which they show the main lines of their approach, illustrating the systematic implementation of Grafcet in IEC 61499. 


\section{WHY A TRANSLATION TO THE C LANGUAGE?}

The Grafcet is a logical automatism description formalism, allowing to express competing processes. If a language has to translate it, it must be preferably parallel (Remaki.Z and al, 1994) and / or real time. In the choice of such a language, a factor such as the duration of the task's installation (or the task's switching time) is decisive. C/Unix (Stevens. W. R, Rago, 2004) was chosen, it presents advantages: portability, universality, control by a majority of computer scientists. Its disadvantage lies in the fact that Unix is not quite real time, because its too slow temporal primitives were defined according to the only problems of timeshare. The $\mathrm{C}$ language is located between the high-level languages, which it owns the data and control structures, and the HLMOL (High-Level Machine-Oriented Language) languages: it allows to define fields to bit close and Expanded by Unix libraries, C can provide on uniprocessor machines, the illusion of simultaneous execution of tasks, as shown by functions: FORK, WAIT, SLEEP, KILL(Stevens.W.R, Rago, 2004). The main means of communication between unix processes: pipes (Stevens.W.R, Rago, 2004). Several synchronization means are available under unix. In our study we have retained the WAIT function which constitutes the most basic mean of synchronization, and which makes it possible to synchronize a father process on the termination of its sons. Time management (Stevens.W.R, Rago, 2004) is carried out with the sleep() function : The call to the sleep(n) function suspends the calling process for a duration of $n$ seconds. Since the second time unit is not realistic for many real-time scenarios, we use the macro tempo (), allowing us to do timers in microseconds.

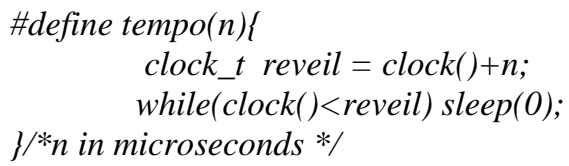

Sleep(1) is then equivalent to tempo(1000000). These timers are used in processes running in parallel and can limit the waiting time of certain events.

\section{SIMULATION SYSTEM DESCRIPTION}

The simulation system mainly consists of two subsystems:

- Grafcet entry : this first subsystem offers two possibilities :

- Graphical input: based on a graphical editor, allows any Grafcet to be entered graphically, and outputs an image data structure of the entered Grafcet.

- Text entry: in practice, rarely used, except for maintenance purposes; it allows to enter the Grafcet as a text, so requires a text analyzer that outputs, for the same Grafcet, the same data structure as the graphical editor receiving the corresponding Grafcet.

- Translation of the Grafcet: this second subsystem exploits the data structure resulting from the entry, and translates the Grafcet entered in the chosen target language, here C; two steps are possible:

- Interpreter / simulator: A program that, according to the data structure, step by step executes the appropriate $\mathrm{C}$ sequences.

- Generator: program that creates a complete $\mathrm{C}$ code image of all the Grafcet; the execution of this code constitutes the simulation of the automatism specified by the Grafcet (as long as this code is not configured according to real $\mathrm{I} / \mathrm{O}$ ).

In both cases, the translation requires, first of all, to find the generation schemes from Grafcet to $\mathrm{C}$. 


\section{OUR APPROACH}

\subsection{Definition}

To find the translation schemes of the Grafcet in C language, consists of finding for each basic element of the Grafcet, a scheme of program (macro, procedure, ...) in $\mathrm{C}$ which has the same semantics. The elements of the Grafcet are: the simple transition, divergences (AND, OR), convergences (AND, OR), the stage and the macro stage.

\subsection{Preliminary Study}

Let's consider the following scenario:

- $\quad$ rotating bar at a position $\mathrm{x}, \mathrm{y}$ of the screen (successive display in $\mathrm{x}, \mathrm{y}$ of characters $-, /,||,,-, /, \ldots$ ),

- $\quad$ in case of no overflows, pressing the $\downarrow, \leftarrow, \rightarrow, \uparrow$ keys causes the bar to move down, left, right and up, respectively,

- If the limits are exceeded, pressing the preceding characters will sound an audible signal and the bar will remain rotating in the same place.

- $\quad$ Pressing the character ' $q$ ' stops the scenario,

- $\quad$ Pressing any other character is ineffective.

Figure 1 shows the Grafcet describing the previous scenario.

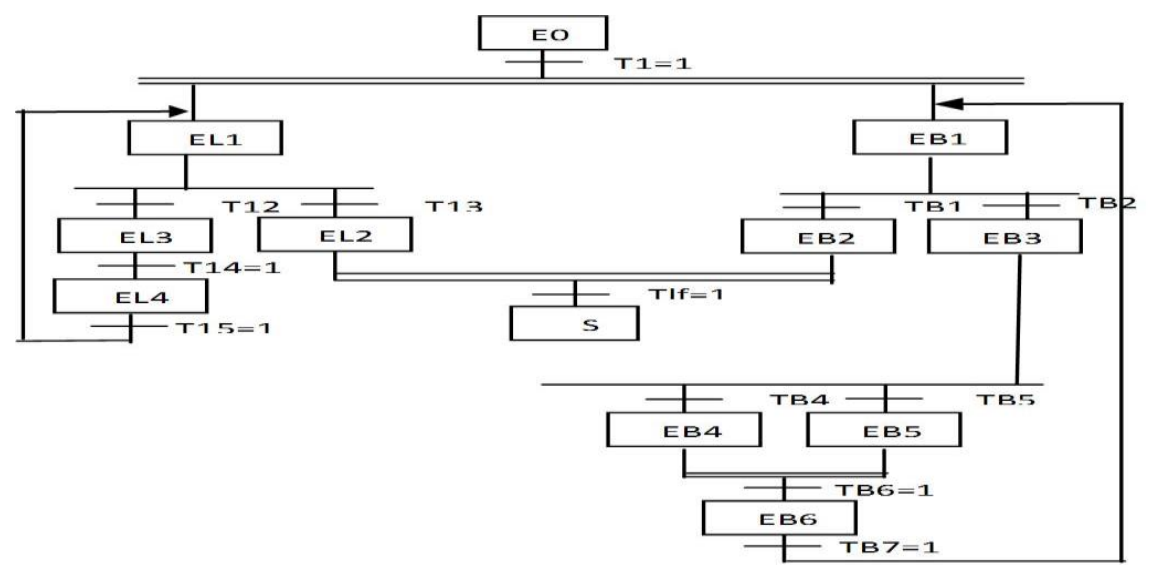

Figure 1. Grafcet associated with the rotating bar

nb. Let EB and TB be the stages and transitions of the rotating bar branch, and EL and TL are the stages and transitions of the reading characters branch.

(1) Stages

E0: initial stage, beginning of the program.

EB1 : rotating bar.

EB2 : end of the rotating bar process.

EB3 : Test of the character read in the pipe.

EB4: audible alarm.

EB5: moving the bar.

EB6: rest (sleep).

EL1: read and test the read character.

EL2: sending a KILL and exit.

EL3: write $\downarrow, \leftarrow, \rightarrow, \uparrow$ in the pipe.

EL4: rest (sleep).

$\mathrm{S}$ : end of the scenario.
(2) Transitions

T1: pressing any key to start both processes.

TB1: reception of a KILL signal.

TB2: read $\downarrow, \leftarrow, \rightarrow, \uparrow$ in the pipe.

TB4: overcoming limits.

TB5: no overflows.

TB6: $=1 ;$ TB7 $=1$

TL2: read $\downarrow, \leftarrow, \rightarrow$ or $\uparrow$ from the keyboard.

TL3: reading of the ' $q$ ' character of the keyboard.

TL4 = 1; TL5 = 1; TLF = 1 . 


\subsection{Principle of the Image Program}

Once the program is launched, two processes are created and launched simultaneously. Note proc1 and proc2 these two processes. Proc1 is associated with the rotating bar, or standalone process. Proc 2 is associated with the movement of the bar, or process under external influence. It is clear that at one point, the two processes proc 1 and proc 2 must act simultaneously on the coordinates $(x, y)$ of the bar. A first solution is to create a critical section within each process to achieve mutual exclusion of both accesses (Raynal.M, 2013). The second would be to allow effective access to a single process, such as proc1, which will rotate and move the bar, proc2 reads the characters $\downarrow, \leftarrow, \rightarrow, \uparrow$ considered as the move commands of the bar, and send them through a pipe to proc1 which will use them to move the bar in the desired direction, the end of the scenario will take place when proc 2 reads the character ' $q$ ' key. We present in the table 1, the general processing algorithms of proc1 and proc2.

Since the program is modeled on the Grafcet, we make it a basis to deduce the basic translation schemes from the Grafcet into C. These schemes have been extended to the case of industrial processes where the system entries can be numerous and simultaneous and especially in real time; therefore push buttons and sensors have been integrated.

Table 1. The Parallel running of Proc1 and Proc2

\begin{tabular}{|c|c|}
\hline Proc1 & Proc2 \\
\hline $\begin{array}{l}\text { At each position of the bar: } \\
\text { - display the bar } \\
\text { - look in } \mathrm{p}[0] \text { if a character is placed in the pipe. } \\
\text { - If yes the character is stored in cc } 1 \text { and tested. } \\
\text { - If exceeding the limits, warning } \\
\text { sound, otherwise move in the desired } \\
\text { direction. } \\
\text { - rotation of the bar. }\end{array}$ & $\begin{array}{l}\text { - Reads a cc character from the keyboard and test it } \\
\text { - If cc ='q' then sends a SIGKILL to proc1 then EXIT. } \\
\text { - If cc ='\#' ,' ', '!' or '"' then places cc in the inlet p[1] } \\
\text { of the pipe; } \\
\text { - If cc is other, ignore cc. }\end{array}$ \\
\hline
\end{tabular}

\subsection{Grafcet-C Translation Schemes}

\subsubsection{Simple Transition}

The simple transition is an external event, which can arise at any time from a sensor, a push button, etc ... To simulate it three cases arise:

\section{(1) Keyboard}

$$
c c=\operatorname{getch}()
$$

\section{(2) Order Box}

Let boitcom the port address of the command box, reading 8 to 16 digital inputs. By assimilating all the bits to zero at the absence of an entry, we will have the scheme: while(! (*boitcom )) sleep $(0)$;

These diagrams will be taken up and developed when a configuration language will cover the essential cases concerning the real-time management of arbitrary devices, which will be part of a realistic simulation; meanwhile, the input from the control box can be simulated by programming the keys of the keyboard, we associate in this case a sequence of bits: command [0] ... command [7], with command [i] $=1$ hence the receptivity $i$ is true; and command $[i]=0$ for $i=1, \ldots, 7$ means that no receptivity is true. 


\section{(3) always occurrent}

$\mathrm{t}=1$; which means that the sum of the internal and external receptivity conditions is 1 ; in this case, we will generate a comment: $\quad / * t=1 * /$ presumed zero cost, and facilitating research for development.

\subsubsection{Divergences}

Case of a transition followed by several stages

\section{(1) AND Divergence}

while $(! t)$ sleep $(0)$;

Rep $=$ forkn(tab_fonc,nproc,tab_pid);

Repl=wait \&status);

while((rep1!=-1) wait(\&status);

Where,

Tab_fonc is an Array of pointers to functions performed in the context of each process, Nproc is the number of the created processes, Tab_pid is pid chart of the created processes and Tab_fonc[i] is the pointer to the function executed by the process with the pid tab_pid[i]. the forkn () function based on the fork() function, it is used to create multiple processes (Stevens.W.R, Rago, 2004).

\section{(2) OR Divergence}

\section{- Exclusive OR}

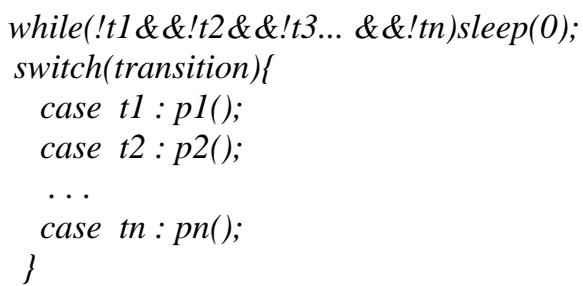

\section{- Inclusive OR}

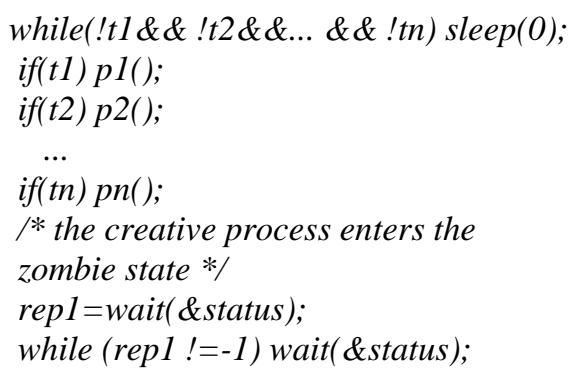

With pi(): the process creation procedure $\mathrm{i}$. There is a boot of new paths.

We are here in the classical case of an input form, likely to have two images: A general image but expensive (competition diagram), or An effective image applicable under certain conditions: here, the alternative scheme, applicable if and only if the transitions are disjoined two by two.

In a generator, the evaluation of this question is the subject of a decision procedure, which in case of difficulties may substitute, the absolute criterion above, one or more sufficient conditions easier to evaluate. (For example here, if two transitions occur as products of elementary conditions, they are disjoint if the same condition is present in the two transitions in opposite forms, such that: $\mathrm{T} 1=\mathrm{x} \mathrm{y} \mathrm{z}$ and $\mathrm{t} 2 \mathrm{x} \mathrm{y} \mathrm{z}$. 
In the case of expectations with time-out, there is divergence with 2 issues, one of which carries the receptivity "event" and the other a receptivity "time limit"; since this can only be performed in excess, it is normal to consider the two as disjoint (in case of conjunction the event is considered to have happened after the prescribed duration). The quality of the decision procedure thus directly governs the quality of the code which may be inaccurate, correct but heavy, correct and effective, and even optimal for a perfect decision procedure. On the other hand, the generation with the divergences must be in agreement with the generation with the convergences.

\subsubsection{Convergences}

\section{- AND Convergence}

Two cases arise: a) pi have, as origin, the same divergence: the synchronization is done by WAIT. b) pi do not have the same origin: in this case, synchronization is mandatory. We describe below its principle.

Suppose for each father process, synchro a global variable.

i. Initially: synchro = number of incoming branches ;

ii. Once finished, each process simulating an incoming branch, must access synchro and decrement it.

iii. When synchro becomes null implies the end of all branches. It is clear that the access to synchro may be simultaneous; access to synchro must therefore be a critical section within each process; you must use either semaphores or locks (Raynal.M, 2013).

\section{The Method Used}

Choose a process, which we will call coordinator; take the one that simulates the most left incoming branch. When finished, the coordinator has to perform Algorithm 1.

Once it has finished, each of the other processes associated with other branches shall perform Algorithm 2:

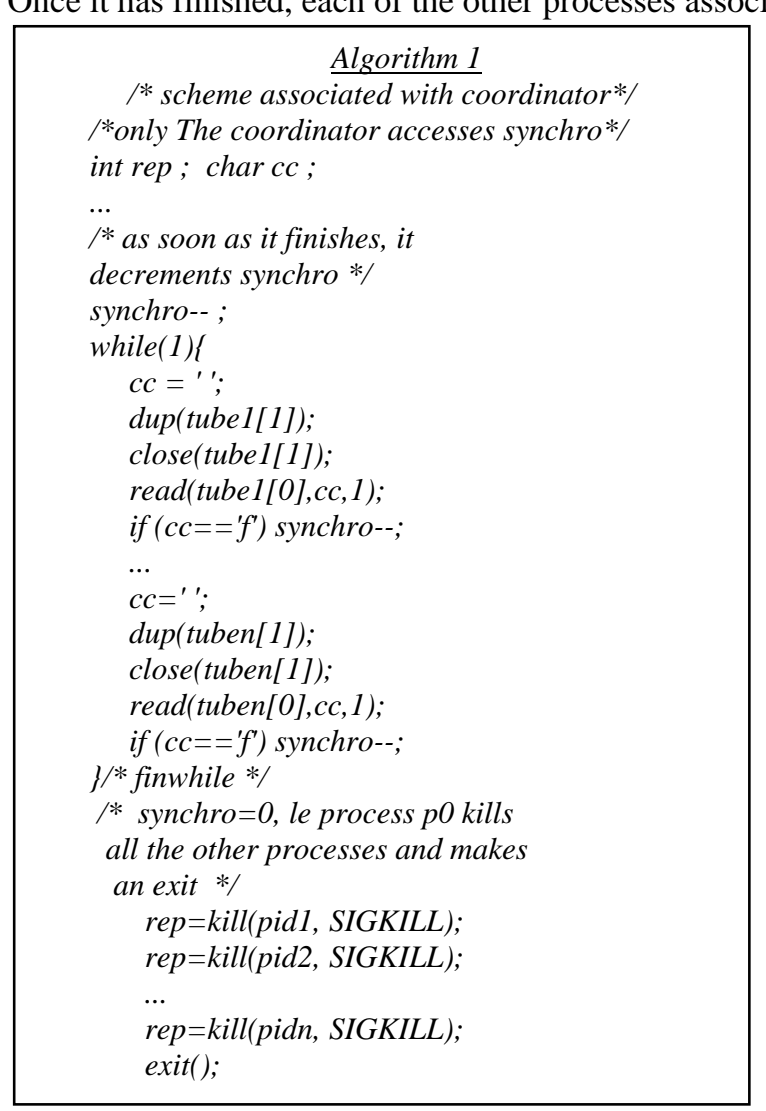




\section{- OR Convergence}

i. Exclusive OR: In practice, this case comes down to the simple transition.

ii. Inclusive OR: This case is discussed in the same way as AND convergence.

\subsubsection{The Stage}

The stage executes, within a process, and can be simulated by a message display specifying it, possibly a time-dependent timer associated with it, encapsulated in stage() procedure.

Void stage(num_stage, duration)

printf("stage \% $\bar{d}$ ",num_stage);

tempo(duration);

\subsubsection{The Macro Stage}

The macro-stage is translated using a procedure that is an image similar to the main program, because it is, in fact, a sub-Grafcet; obviously militates for a recursive generation.

\section{THE NECESSARY CONFIGURATION FOR SUCH A SYSTEM}

The necessary configuration may be: a) Unix or Linux system or any multitasking. b) A graphic screen for entering the Grafcet. c) As input device a keyboard and, if possible, a box of commands. d) As effectors: LEDs, bulbs, effectors.

Simulation process: The screen is divided into two windows: FEN1, for the drawing of the currently active Grafcet (command part). FEN2, specific to the messages illustrating the actions performed by the operative part. Initially, the Grafcet is animated from the initial situation; the arrival of the transitions of the keyboard or of the control box causes the evolution of the Grafcet, ie activation of the new, stage or stages (following the transition), and deactivation of the stage or stages, preceding. The active stages will be highlighted in FEN1, their associated messages in FEN2. The use of the order box is because it allows you to make several entries at once, a case that can be tested for OR divergences, transitions whose branches run in parallel. This will be a realistic simulation.

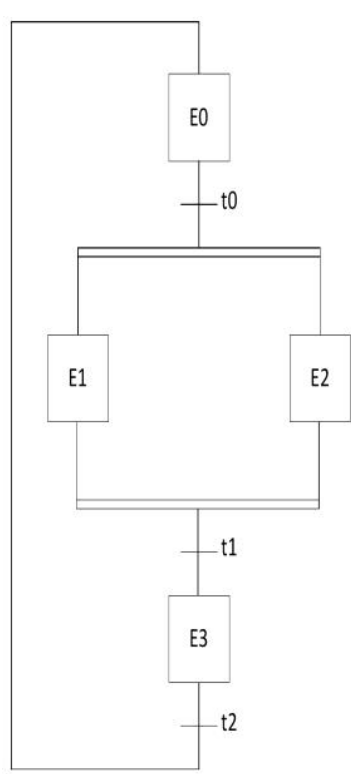

a) The Grafcet

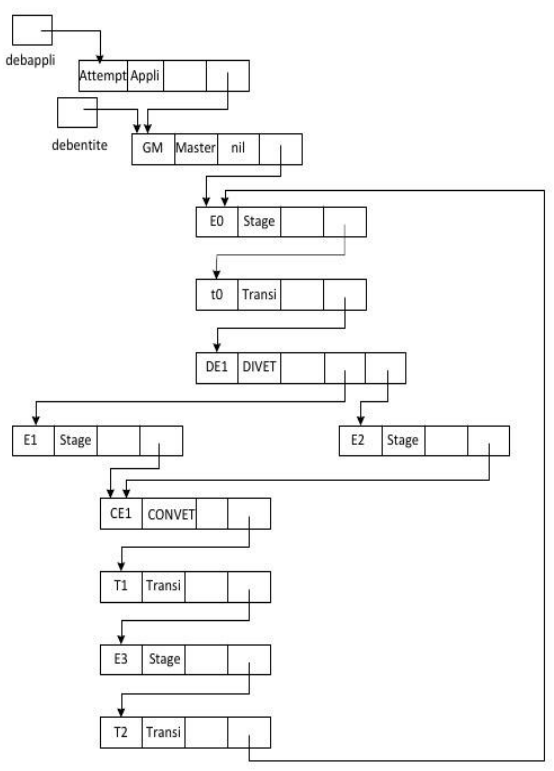

b) The internal form

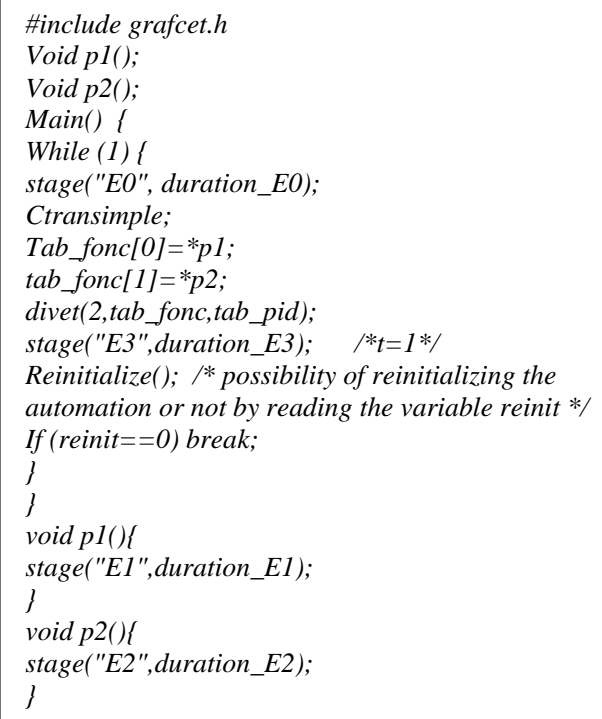

Figure 2. From the Grafcet to the $\mathrm{C}$ program 


\section{CONCLUSION}

The method followed, allows to translate many Grafcets, by composition of the most elementary elements. In practical terms, we have established a Grafcet.h library of translation schemes. This library has been tested on examples and is independent of the supposed internal form, it takes into account the automatisms described by a unique Grafcet; and can be extended to explicit macros and procedures relating to forcing processing, used in the case of a hierarchy of Grafcets. Starting from a graphic editor or specialized analyzers, our schematics will constitute a basis for the automatic production of $\mathrm{C}$ programs whose execution will behave according to the Grafcet treated. As a result, once the program has been configured, tested and validated, the object code can be implemented on a microcontroller of the actual control system, in particular by explaining everything that pertains to the execution configuration.

Our program can be widely used, because of the simple configuration, hardware and software, it requires: for instance, for educational purposes, for someone (high school student) who wants to learn the Grafcet formalism; or by an automation engineer in his research laboratory who wishes to validate the specifications of his automatism. All that is needed is a computer staff and a linux environment.

From a technical point of view, the adopted internal form can be used for automatic archiving of Grafcets. The archiving of Grafcets is a crucial task for automation engineers who still want to keep track of their initial designs of their automation systems in order to modify specifications when carrying out maintenance operations; or the extension of the functions of the automation to improve the efficiency of their installations. Our perspective in this work, is the translation of the Grafcet in Promela / SPIN. This time, our goal will be mostly formal verification of automation before its implementation on real installations.

\section{ACKNOWLEDGMENT}

Our tribute goes to Mr Louis Frécon, who was a professor at INSA / Lyon, and who died on November 11, 2018; for his competitions during the realization of this work.

\section{REFERENCES}

Benaouda.N., (2017). Modélisation et Gestion des Activités de Maintenance dans les systèmes de Production, doctoral thesis in computer science, SETIF-1 University, Setif, Algeria.

Blanchard. M, (1994). Comprendre, maîtriser et appliquer le grafcet, Cepadues, Toulouse. David, R. and Alla.H., (2010). Discrete, continuous and hybrid Petri nets. Springer-Verlag, Berlin.

Gonzalez.E, Marichal.R \& Hamilton.A, (2016). Ontology-based approach to Basic Grafcet formalization, Journal of the Chinese Institute of Engineers, Taylor \&Francis, Vol. 39, No. 8. pp. 946-953.

Julius.R, and al (2017). Transformation of GRAFCET to PLC code including hierarchical structures, Control Engineering Practice, Elsevier, Vol. 64, pp. 173-194.

Julius.R. and al. (2019). A model-driven approach for transforming GRAFCET specification into PLC code including hierarchical structures, in IFAC- PapersOnLine, Vol.52, No.13, pp. 1767-1772.

Miguel-Escrig.O and al (2020). Distributed implementation of Grafcets through IEC 61499. Proceedings of 202025 th IEEE International Conference on Emerging Technologies and Factory Automation (ETFA), Vienna, Austria, 2020, pp. 402-409.

Raynal.M, (2013) Concurrent Programming - Algorithms, Principles, and Foundations, springer.

Reeb.B., Chèze.C., (2011). Développement des grafcets. Des machines simples aux cellules flexibles, du cahier des charges à la programmation, 2nd edition, ELLIPSES MARKETING.

Remaki.Z, and al (1994). Schémas de traduction grafcet Occam2, 3rd Maghreb Congress on Artificial Intelligence and Software Engineering, Rabat.

Stevens.W.R, Rago.S.A., (2004). Advanced Programming in the Unix Environment, 3rd edition. The Addison-Wesley Professional Computing Series. 